\title{
Computational Method Investigation of Solid Ducted Rocket
}

\author{
Wei Wang ${ }^{\text {a }}$, Gang Zhang ${ }^{\text {b,* }}$, Jun-De Han ${ }^{\text {b }}$, Wei Ma ${ }^{\text {b }}$ \\ ${ }^{a}$ Jiangnan Design Institute of Machinery \& Electricity, Guiyang 550100, China \\ ${ }^{b}$ Systems Engineering Institute of Sichuan Aerospace, Chengdu 610100, China
}

Received: 26 March 2018; Accepted: 07 October 2018; Published: 08 January 2019

\begin{abstract}
The Computation method of Solid Ducted Rocket (SDR) is a complex problem. It needs to effectively solve the two mixing and combustion processes, and is also affected by many factors such as the overall scheme and propellant type. In order to find a suitable method, physical model, simplified hypothesis, control equation, turbulence model, combustion model, etc. were investigated. Subsequently, calculations were carried out based on the Vanka model, and finally the results of pressure, temperature and combustion efficiency were obtained. The results show that the proposed method is practicable, and the accuracy and efficiency are ideal. Combustion efficiency is only $77.4 \%$, and the Vanka model must be further improved by the air and gas intake modes. The results can provide reference for relevant research.
\end{abstract}

Index Terms: Computational method, solid ducted rocket (SDR), Vanka model.

(C) 2019 Published by MECS Publisher. Selection and/or peer review under responsibility of the Research Association of Modern Education and Computer Science.

\section{Introduction}

Solid Ducted Rocket (SDR) is a new type of power plant. It is an air breathing engine that works by using ram air to meet oxygen demand, thereby greatly increases the specific impulse of the propellant and reducing the mass of the engine [1]. SDR has many advantages and has been widely used in many equipment fields, including ground-to-ground missiles, ground-to-air missile, air-to-air missiles, etc. However, the use of ram air also brings many difficulties, such as air intake design, ballistic planning and engine performance, and so on. Among the current challenges, high-performance engine design is one of the key points and difficulty of research. As we all know, the experiment's advantage is that the data are accurate, but it also has many shortcomings such as large workload, long cycle, and high cost, and it is also subject to testing techniques and other factors. In order to quickly promote the research process, the most common method in the actual work is

* Corresponding author.

E-mail address: kevin.cjwong@hotmail.com 
the numerical calculation. Researchers have done a lot of work on the accuracy and efficiency of numerical methods, and have achieved a series of results $[2,3,4]$. On the whole, the focus of numerical method research covers control equations, turbulence models and combustion models.

At present, there are three methods of the numerical problems, direct numerical simulation (DNS), the large eddy simulation (LES) and the Reynolds averaged Navier-Stokes equations (RANS). Because the computational resources required and time consuming are huge for DNS and LES, and only the RANS model can be applied in practical projects. The discrete ways of Navier-Stokes $(\mathrm{N}-\mathrm{S})$ equations can be used in a variety of methods, including finite volume method, finite element method, etc. Lagrangian equations are widely used for the calculation of discrete terms, and it also considers the effects of vortices.

For the analysis of turbulent motion, the current method commonly used in engineering calculations is to introduce a turbulence model to close the Reynolds equation, which is the RANS method. There are various turbulence models, which can be divided into zero equation, one equation and two equations according to the number of equations.

Combustion models include gas-phase combustion models, solid particle ignition and combustion models. For gas-phase combustion simulation, premixed combustion or non-premixed combustion model can be used, which needs to be determined according to actual conditions. In contrast, solid-phase combustion simulations are complex and usually involve two stages of ignition and combustion. Among the ordinary particles, the combustion of boron particles is the most complicated, and the research is gradually deepening [5].

Numerical methods should be analyzed before the practical application, and the key is accuracy verification. But different physical models have different numerical methods. When the experimental data are incomplete, the calculation results are difficult to be accepted. In this paper, the author attempts to investigate a highconfidence numerical method for the Vanka model, and the research results provide reference for subsequent related research.

\section{Computation Method}

In this paper, the computation method consists of six parts, namely physical model, hypothesis, governing equation, turbulence model, combustion model, calculation settings, respectively.

\subsection{Physical Model}

The main parameters of the Vanka model are given in Fig.1. [6]. It can be seen that diameter and length of the secondary combustion chamber are $0.1524 \mathrm{~m}$ and $0.8636 \mathrm{~m}$, separately. There are two intakes. The angle of the intake is 45 degrees, and the axis width is $0.06985 \mathrm{~m}$, and the circumferential width is $0.0508 \mathrm{~m}$. The distance between head and the inlet is $0.0508 \mathrm{~m}$. The fuel nozzle diameter and nozzle critical diameter are $0.01143 \mathrm{~m}$ and $0.064 \mathrm{~m}$, separately.

\subsection{Hypothesis}

To simplify the calculation, this paper makes the following hypothesis [7]:

- Steady flow. In the actual process of flight, inhalation air flow rate is subject to the variation in the flight speed, altitude, attack angle and sideslip angle. It also led to changes in chamber pressure and the air fuel ratio of the combustion. At the same time, an unstable phenomenon happened in the process, so the combustion chamber flow is unsteady in the strict definition. But in transient stability condition, this hypothesis is reasonable as the balance speed is far greater than that of external factors. It is also beneficial to optimization of computing time and efficiency. 


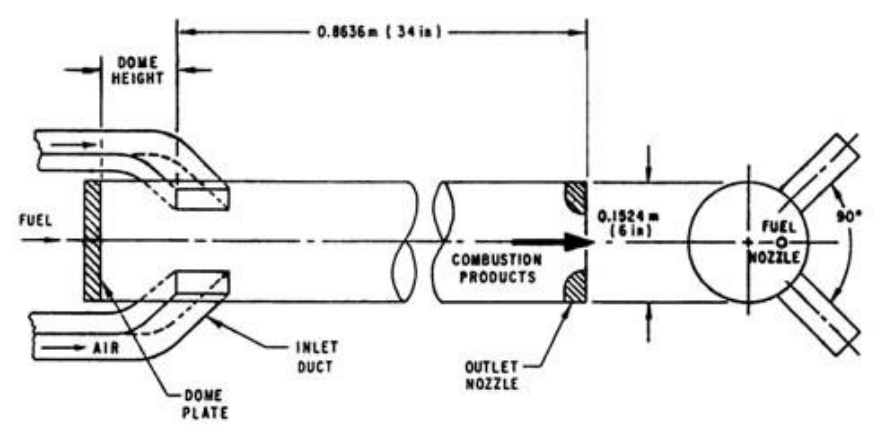

Fig.1. Calculation Structure

- Ideal gas. The combustion chamber gas is assumed to be the ideal gas, and meet with state equation $p=\rho R T$. But $\mathrm{R}$ is the average of gas, oxygen and products. In other words, all components in accordance with the state equation of the ideal gas

- Insulated wall. It is isentropic adiabatic flow, and there is no heat exchange between the flow field and environment.

- Gas gravity forces can often be ignored because of the smaller density, so the influence of body force such as air gravity also does not consider. The erosion effect of combustion also does not take into account in the computation method.

\subsection{Governing Equations}

\begin{tabular}{|ll|}
\hline Nomenclature \\
$\mathrm{Q}$ & conserved quantity \\
$\mathrm{n}$ & normal vector \\
$\mathrm{F}$ & flux vector \\
$\mathrm{Y}_{\mathrm{p}}$ & mass fraction of any kind of product \\
$\mathrm{Y}_{\mathrm{R}}$ & mass component of any kind of product \\
\hline
\end{tabular}

The three laws of fluid motion are conservation of mass, momentum and energy conservation. Based on the three laws, N-S equations, which are the most complete form of the continuum flow, is established by using the mathematical description of fluid motion under considering the fluid viscosity, thermal conductivity and other factors $[4,8]$. The conservation integral form of N-S equations in Cartesian coordinates as showed in Eq 1.

$$
\frac{\partial}{\partial t} \iiint_{\Omega} Q d V+\oiint_{\partial V} F \cdot n d s=0
$$

\subsection{Turbulent model}

The fluid flow can be divided into two kind states, laminar and turbulent. When the Reynolds number is enough in the viscous fluid, the flow will lose its stability and the state will change from laminar to turbulent. It is well known that the gas flow and burn in the combustion chamber are the turbulent state. There are largescale recirculation zones and vortices in the flow field, so that the diffusion of momentum and other scalars is significantly enhanced throughout the field. Therefore, the choice of a suitable turbulence model is one of the 
key steps in the computation model. The $\mathrm{k}-\omega$ model has a wide application range and precision in the near-wall free flow. In the k- $\omega$ SST model, the shear stress transport mode uses the Wilcox k- $\omega$ mode in the near wall, and uses the $k-\varepsilon$ mode in the free shear layer and the boundary layer edge. The turbulence model in this paper uses k- $\omega$ SST, and the turbulent energy transport equation and the turbulent ratio dissipation rate equation are shown in Eq 2 and Eq 3, respectively.

$$
\begin{aligned}
& \frac{\partial \rho k}{\partial t}+\frac{\partial}{\partial x_{j}}\left[\rho u_{j} k-\left(\mu+\sigma_{k} \mu_{t}\right) \frac{\partial k}{\partial x_{j}}\right]=\tau_{t i j} S_{i j}-\beta^{*} \rho \omega k \\
& \frac{\partial \rho \omega}{\partial t}+\frac{\partial}{\partial x_{j}}\left[\rho u_{j} \omega-\left(\mu+\sigma_{\omega} \mu_{t}\right) \frac{\partial \omega}{\partial x_{j}}\right]=P_{\omega}-\beta \rho \omega^{2}+2\left(1-F_{1}\right) \frac{\rho \sigma_{\omega 2}}{\omega} \frac{\partial k \partial \omega}{\partial x_{j} \partial x_{j}}
\end{aligned}
$$

In the formula, end of the right representative cross diffusion, and the remaining parameters specific forms can be found in the reference $[4,8]$.

\subsection{Combustion Model}

The purpose of this paper is to investigate numerical methods. Many different simplified or approximate ways have been proposed. In combustion model, ethylene are provided in calculation by properties of matter representativeness analysis, and the reaction between the fuel and the air is

$$
\mathrm{C}_{2} \mathrm{H}_{4}+3 \mathrm{O}_{2}=2 \mathrm{CO}_{2}+2 \mathrm{H}_{2} \mathrm{O}
$$

In order to combustion simulation, reactants parameters should be pre-given, which are shown in Table.1.

Table 1. Reactants Parameters

\begin{tabular}{lll}
\hline Parameters & Air & Ethylene \\
\hline Molecular weight & 25.36 & 28.00 \\
Temperature $(\mathrm{K})$ & 556 & 556 \\
Specific heat at constant pressure $\left(\mathrm{J} . \mathrm{K}^{-1} \cdot \mathrm{kg}^{-1}\right)$ & $927.00+2.58 \mathrm{~T}+3.82 \times 10^{-5} \mathrm{~T}^{2}$ & $404.00+4.36 \mathrm{~T}-1.35 \times 10^{-3} \mathrm{~T}^{2}$ \\
Burning heat $\left(\mathrm{J} \cdot \mathrm{kg}^{-1}\right)$ & - & $4.895 \times 10^{7}$ \\
equivalence ratio & - & 15.58 \\
\hline
\end{tabular}

The combustion product molecular weight is 25.55 , and the chamber pressure is $5.0 \times 10^{5} \mathrm{~Pa}$.

The reaction rate is described by the eddy dissipation model, material $i$ production rate in reaction $r$ is given by Eq $5[9,10]$.

$$
R_{i, r}=\min \left\{v_{i, r}^{\prime} M_{w, i} A \rho \frac{\varepsilon}{k} \min _{R}\left(\frac{Y_{R}}{v_{R, r}^{\prime} M_{w, R}}\right), v_{i, r}^{\prime} M_{w, i} A B \rho \frac{\varepsilon}{k} \frac{\sum Y p}{\sum_{j}^{N} v_{j, r}^{n} M_{w, j}}\right\}
$$


In the above formula, $A, B$ is the calculation empirical parameters, the values are 4 and 0.5 in this paper, respectively.

\subsection{Mesh And Boundary Settings}

Grid division rationality is another influence factor of calculation accuracy. This paper uses the size function of the GAMBIT control mesh generation [11]. The structured grid is employed in most areas and grid refinement technology is selected for rather change part. In the end, the structure divided into 115623 points, 449404 faces, 172329 cells, and the final grid as showed in Fig.2.

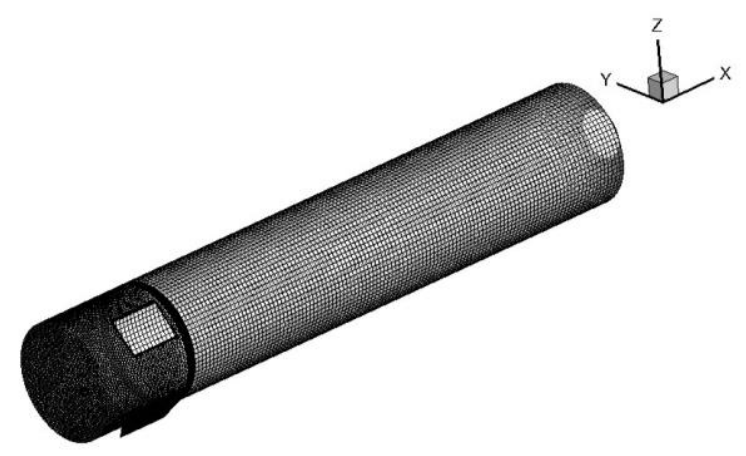

Fig.2. Computational Grid

To solve the equations of combustion, the boundary conditions must to be given, and its rationality will directly determine the convergence and accuracy. According to the working state of the engine and the physical characteristics, boundary conditions are determined as follows:

- Wall condition. The wall is no slip $(\bar{u}=0)$, adiabatic $(\partial T / \partial \bar{n}=0)$, non-catalytic $(\partial C / \partial \bar{n}=0)$, and also ignores the change of total pressure at the surface normal direction of the first layer $(\partial P / \partial \bar{n}=0)$.

- Mass flow condition. There are two entrance boundaries, the gas and air, respectively. The fuel enters the chamber at a certain flow rate and direction. The flow rate mainly depends on the fuel surface retreating rate of the propellant. The required parameters for the calculation are: mass flow rate, total temperature, total pressure, flow direction, etc. The air comes from the inlet outlet, so the mass flow boundary is still selected for the calculation.

- Outlet condition. The outlet is end of the expansion section of the nozzle. So all the boundary parameters can be obtained by the numerical extrapolation because the supersonic flow in this section. If the outlet pressure is less than that of the environment under the subsonic condition, boundary pressure is equal to the external pressure and its value determined through the design height interpolation 'American Standard Atmospheric 1976'.

\section{Result Analysis}

It's necessary to initialize the flow field parameters when the time correlation method is used to calculate the stationary problem. In theory, the flow will tend to a certain state regardless of the initial condition, but in the practical application, it will directly affect the convergence and efficiency. In this paper, the flow field is initialized by entrance conditions at the beginning of calculation, and other working conditions can choose the representative results in order to accelerate the convergence speed and reduce the calculation time. 
The pressure distribution in the computation domain is illustrated in Fig.3. It can be observed that the value is about $500000 \mathrm{~Pa}$, and is basically the same as the set value. This shows that the calculation settings used in this paper are correct. Pressure distribution is evenly except the near the inlet, which located at the head of the chamber and its pressure is increased cause by air injection. Gas expansion has caused pressure sudden drop in the nozzle area and the outlet pressure average fall to $346995 \mathrm{~Pa}$. The calculation results are basically consistent with the theoretical analysis.

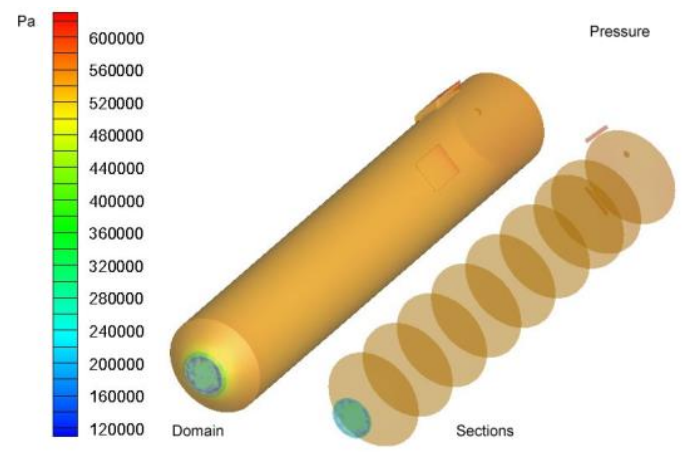

Fig.3. Pressure Distributions

The average values of pressure and Mach number at various x-coordinates are listed in Table 2 . The results also verified the above conclusion. At the same time, it's also can be noted that average pressure of the cylindrical section is drops slowly along the flow direction. Theoretically, since the flow cross-sectional area has not changed, the value should not change. Therefore, the cause of the above phenomenon may be a calculation error. This result also demonstrates that the chamber pressure is the largest, and chamber strength should be guaranteed to meet the design requirements.

Table 2. The Average Values of Pressure and Mach number at Various X-coordinates

\begin{tabular}{ccc}
\hline x-coordinate $(\mathrm{mm})$ & Average pressure $(\mathrm{Pa})$ & Average Mach \\
\hline-5 & 565885.19 & 0.77271 \\
50 & 559993.00 & 0.16134 \\
150 & 548099.69 & 0.14157 \\
200 & 546058.81 & 0.14236 \\
300 & 544948.38 & 0.14257 \\
400 & 544634.63 & 0.14085 \\
500 & 544473.13 & 0.13934 \\
600 & 544316.88 & 0.13832 \\
700 & 544162.19 & 0.13770 \\
800 & 544099.69 & 0.13734 \\
863 & 346995.31 & 1.05601 \\
\hline
\end{tabular}

The flow speed in the combustion chamber is subsonic, and its change is very small. The flow in the nozzle is gathering speed and meets congestion state in the throat, which showed in Fig.4. This phenomenon is in agreement with the trend of cross-section changes. It is necessary to pay attention to the scouring effect of the high-speed airflow on the nozzle, and to rationally design the expansion ratio of the nozzle to ensure the optimal performance of the domain. 

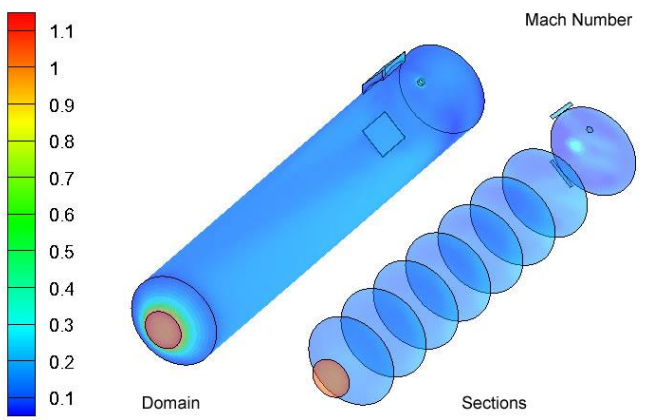

Fig.4. Mach number Distributions

The velocity vector in the $\mathrm{z}=0$ plane is given in Fig.5. As can be seen from the figure, the gas is turned on the upper side of the $\mathrm{Y}$-axis, because the speed difference between the gas and the air is small, and the air inlet and the fuel inlet are located on the same side. This phenomenon will cause a certain impact on combustion between $\mathrm{C}_{2} \mathrm{H}_{4}$ and oxygen. Specifically, this phenomenon promotes an increase in combustion efficiency on the one hand, but it also easily causes uneven combustion. Specifically, this phenomenon is advantageous for promoting combustion and improving energy conversion efficiency, but it is also liable to cause uneven combustion [12]. In actual design, multiple factors should be considered comprehensively.

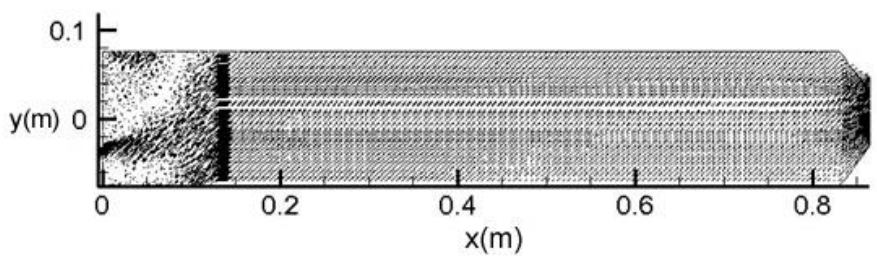

Fig.5. Velocity vector in the $\mathrm{z}=0$ plane

To illustrate the above result, temperature distribution of the domain and the plane in $\mathrm{z}=0$ are shown in Fig. 6 and Fig.7, respectively. It was clear from figures that temperature distribution is clearly uniform caused by intake mode. In addition, the high temperature zone is mainly concentrated on the side of the chamber, and the maximum temperature can reach about $2500 \mathrm{~K}$. The reason is that the amount of fuel is large and the combustion is sufficient. This phenomenon has two main effects, one is that the combustion efficiency is low, and the other is that it is difficult to properly prevent heating.

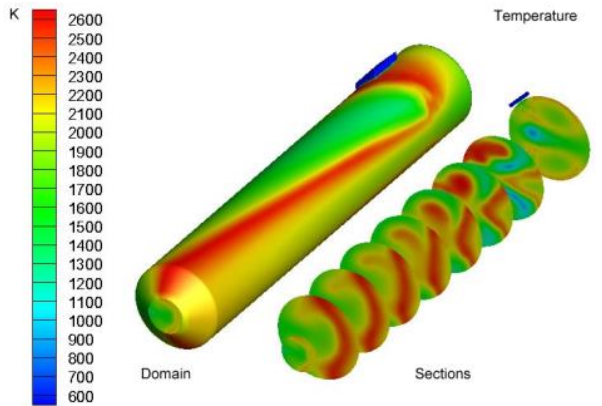

Fig.6. Temperature Distribution 


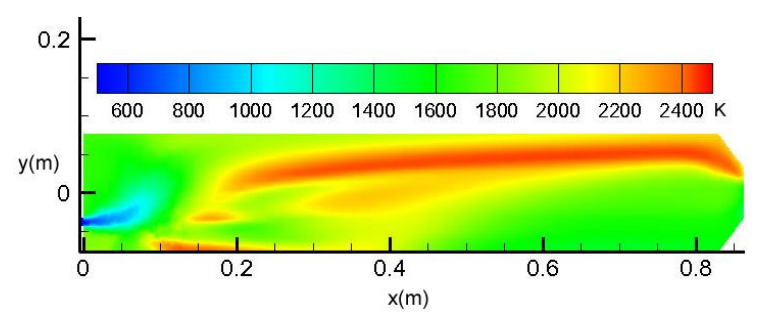

Fig.7. Temperature Distribution in the $\mathrm{z}=0$ plane

The mass fraction distribution of the reactant and main product $\left(\mathrm{CO}_{2}\right)$ is presented in Fig.8 and Fig.9, respectively. It can be seen from the figure that the distribution is uniform, whether it is the reactant or the main product, and the trend is consistent with the previous conclusions. This again proves the correctness of the method.

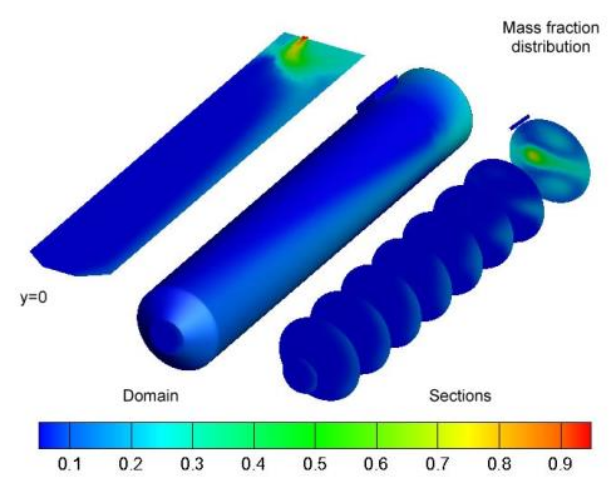

Fig.8. Mass Fraction Distribution of the Reactant

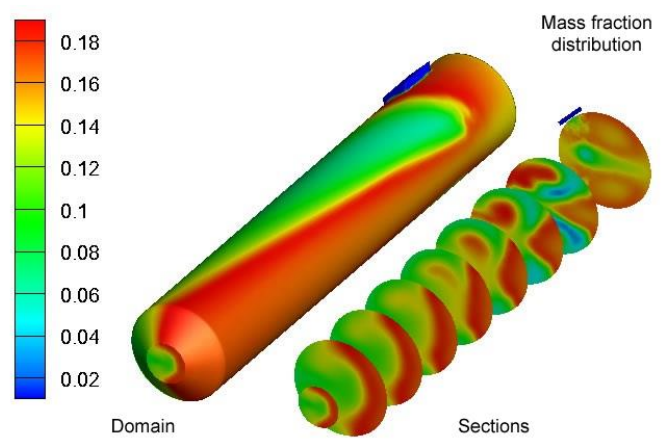

Fig.9. Mass Fraction Distribution of the $\mathrm{CO}_{2}$

Mass fraction of $\mathrm{C}_{2} \mathrm{H}_{4}$ at the center line is shown in Fig.10. It can see that the concentration of the head region is raised to about 0.5 . The main reasons are that the mixing in these areas is not sufficient, and the consumption is less than the effect of fuel injection and diffusion. As the gas flows toward the nozzle, the effect of consumption becomes more pronounced, and the effect of injection and diffusion is gradually weakened. With the combination of the two factors, the $\mathrm{C}_{2} \mathrm{H}_{4}$ will be consumed in an area. In this paper, the reaction is basically completed at the head distance of $200 \mathrm{~mm}$. 


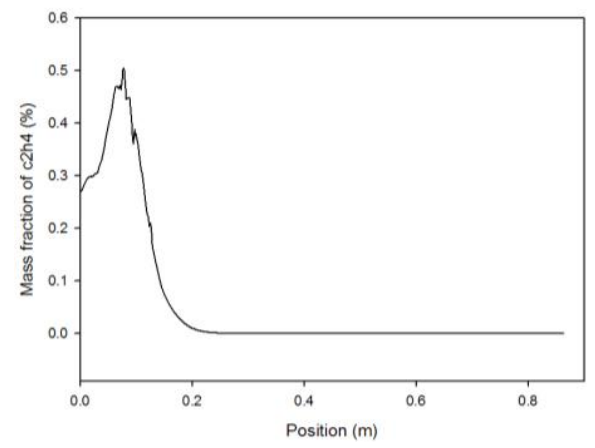

Fig.10. Mass Fraction Distribution of the $\mathrm{C}_{2} \mathrm{H}_{4}$ at Center Line

The combustion efficiency is defined as

$$
\eta_{i}=\frac{m_{i 1}}{m_{i 0}} \times 100 \%
$$

In the formula, the subscript $i$ indicates a reactant or product formula, 0 stand for theory value, 1 represents the actual production

The $\mathrm{CO}_{2}$ is selected, and the combustion efficiency is $77.4 \%$. Current research has shown that efficiency has to be greater than $85 \%$, or even close to $90 \%$. This shows that the design of the Vanka model is not reasonable and requires research on combustion organization technology. The result agrees well with the existing conclusions.

\section{Conclusion}

In this paper, the research progress of the computation method of SDR is briefly described. Then the numerical method is established based on Vanka model. The pressure, temperature and velocity distribution of the example is given. Finally, the combustion efficiency is calculated. The conclusions of this study are as follows:

- The computation method in this paper is correct and effective, and the results can provide reference for related work.

- Combustion efficiency is only $77.4 \%$, which is far below the acceptable level, and the Vanka model must be further optimized for performance.

- The combustion efficiency of the SDR can be improved by optimizing the air and gas intake modes.

\section{References}

[1] Johns Hopkins University.ramjet technology [M]. Beijing: National Defense Industry Press, 1987.

[2] Yan Chao. Computational fluid dynamics method and application [M]. Beijing: Beihang University Press, 2006

[3] Liu Daozhi. Computational fluid dynamics [M]. Beijing: Beihang University Press, 1989.

[4] Anderson J D. Computational fluid dynamics: the basics with application [M]. New York: McGRAW- 
Hill. 1995.

[5] Merill K King.Ignition and combustion of boron particles and clouds [R].AIAA 82-4174,1982

[6] Ma Liangdong,Zhang Jili,Zhang Dingcai.Large eddy simulation of turbulent flow and heat transfer in a rotating rectangular duct [C] .The First International Conference on Building Energy and Environment, 2008, Dalian Liaoling

[7] Tucker, P. K., Menon, S., Merkle, C. L., Oefelein, J. C. \& Yang, V., Validation of high-fidelity CFD simulations for rocket injector design, 44th AIAA/ASME/SAE/ASEE Joint Propulsion Conference and Exhibit, Hartford, CT,2008.

[8] Ferziger J H, Peric M. Computational methods for fluid dynamics [M]. New York: Springer, 2002.

[9] Zhang Hanxin, Shen Mengyu. Calculation of fluid mechanics-difference method principle and application [M]. Beijing: National Defence Industry Press, 2003

[10] Yang, Y., Xing, J. W., Le, J. L. \& Wang, J. N., Effect of turbulent combustion model on simulation of hydrogen supersonic combustion, Journal of Aerospace Power, 23(4):506-516,2008.

[11] Zhu Hongjun, Lin Yuanhua, Xie Longhan. Fluent 12 analysis and Engineering simulation [M]. Beijing: Tsinghua University press, 2011

[12] Mohammad.A, Toshi, F. Joseph.E.L, Influence of Main Flow Inlet Configuration on Mixing and Flameholding in Transverse Injection into Supersonic Airstream, International Journal of Engineering Science, 38(11):1161-1180, 2000.

\section{Authors' Profiles}

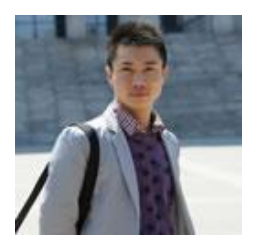

Gang Zhang is working as an engineer at the systems engineering institute of Sichuan aerospace. He holds a master degree of mechanical engineering. His fields of scientific interests are optimization design, structural mechanics.

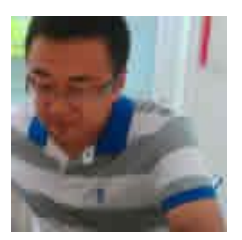

Jun-De Han is working as a senior engineer at the systems engineering institute of Sichuan aerospace. He holds a master degree of energy engineering. His fields of scientific interests are combustion process, energy technology.

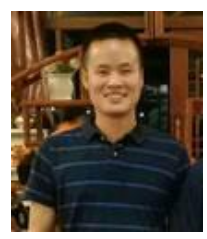

Wei Ma is working as an engineer at the systems engineering institute of Sichuan aerospace. He holds a master degree of fluid mechanics. His fields of scientific interests are optimization design, fluid mechanics.

How to cite this paper: Wei Wang, Gang Zhang, Jun-De Han, Wei Ma,"Computational Method Investigation of Solid Ducted Rocket", International Journal of Engineering and Manufacturing(IJEM), Vol.9, No.1, pp.1-10, 2019.DOI: 10.5815/ijem.2019.01.01 